\title{
A REPRESENTATION OF GENERALIZED INFRAPOLYNOMIALS ${ }^{1}$
}

\author{
BRUCE L. CHALMERS
}

ABSTRACT. A representation theorem is obtained for generalized infrapolynomials on an arbitrary complex point set. Some results on the location of zeros are also presented.

Let $P_{n}$ denote the class of polynomials $\sum_{i=0}^{n} c_{i} z^{i}$ with complex coefficients considered as mappings of the complex $z$-plane $\mathcal{C}_{z}$ into itself. Let $\left\{\mathfrak{Q}^{i}\right\}_{i=1}^{s}$ denote a fixed set of $s$ linearly independent linear functionals on $P_{n}$, and $A=A_{1}, A_{2}, \cdots, A_{s}$ be a fixed $s$-tuple of complex numbers. Then $P_{n}(A)$ will represent the class of polynomials $p(z)$ in $P_{n}$ such that $\mathscr{\complement}^{i} p=$ $A_{i}, i=1,2, \cdots, s$. Further, let $E$ denote a compact subset of $\mathcal{C}_{z}$ containing at least $n-s+2$ points. As in [1] we make the following

Definition. $p(z) \in P_{n}(A)$ is called an infrapolynomial on $E$ with respect to $P_{n}(A)$ if $p(z)$ has on $E$ no underpolynomials in $P_{n}(A)$; i.e., if there exists no polynomial $q(z)$ in $P_{n}(A)$ such that

(1) $|q(z)|<|p(z)|$ on $E \cap\{z ; p(z) \neq 0\}$,

(2) $q(z)=0$ on $E \cap\{z ; p(z)=0\}$.

A polynomial $q(z) \in P_{n}(A)$ such that

(3) $|q(z)| \leq|p(z)|$ on $E$

is called a weak underpolynomial of $p(z)$ on $E$ with respect to $P_{n}(A)$.

Let $e_{z}$ denote the functional which gives point evaluation at $z$, i.e. $e_{z} f=f(z)$.

Haar Assumption I. $\left\{\mathfrak{Q}^{i}\right\}_{i=1}^{s} \cup\left\{e_{z}\right\}_{j=s+1}^{n+1}$ is a linearly independent set of functionals on $P_{n}$ for all choices of $n-s+1$ distinct points $z_{s+1}, \cdots$, $z_{n+1}$ in $E$.

Note 1. Recall that, by assumption, $E$ contains at least $n-s+2$ points. Note that if $E$ were to contain only $n-s+1$ points, $P_{n}(A)$ would contain a unique infrapolynomial, vanishing of course at all the points of $E$.

Received by the editors September 19, 1972 and, in revised form, February 3, 1974. AMS (MOS) subject classifications (1970). Primary 30 A82; Secondary 30 A08.

Key words and phrases. Infrapolynomials, Haar Assumption, representation theorem, location of zeros.

1 This research was supported in part by AFOSR Grant 71-2030 A. 
Note also that if $E$ were to contain $n-k+1$ points, $k \geq s, P_{n}(A)$ would contain a $(k-s)$-dimensional set of infrapolynomials of the form $I_{0}(z)+h(z)$, where $I_{0}$ is a fixed element of $P_{n}(A)$ vanishing on $E$, and $h(z)$ is an arbitrary element of $P_{n}$ vanishing on $E$ and such that $\mathcal{L}^{i} h=0(i=1, \cdots, s)$.

Note 2. In [1] it was shown that under a slightly stricter assumption (namely the Lagrange set $\left\{e_{z_{j}}\right\}_{j=s+1}^{n+1}$ on $n-s+1$ points is replaced by the more general Hermite set of ${ }^{j}-s+1$ derivative evaluations in Haar Assumption I), an infrapolynomial on $E$ with respect to $P_{n}(A)$ has no weak underpolynomials on $E$ with respect to $P_{n}(A)$.

From Theorems 1, 2 and 3 of [4] we immediately obtain the following result.

Theorem 1. If, with respect to $P_{n}(A), p(z)$ is an infrapolynomial on $E$, then $p(z)$ is an infrapolynomial on a set $E_{r}=\left\{z_{s}, z_{s+1}, \cdots, z_{n+r+1}\right\} \subset E$, where $0 \leq r \leq n-s+1$. In particular, if the elements of $P_{n}(A)$ are all realvalued on $E$, then we may take $r=0$.

Haar Assumption II. Given $E_{r}$ as above, $\left\{\mathfrak{Q}^{i}\right\}_{i=1}^{s-1} \cup\left\{e_{z_{j}}\right\}_{j=s}^{n+r+1}$ is a linearly independent set of functionals on $P_{n+r}$.

Note 3. In this assumption $\mathfrak{L}^{s}$ has been removed from consideration.

We will next give our main result which is a representation of infrapolynomials on $E_{r}$ with respect to $P_{n}(A)$. But first we introduce some notation. Let $\left\{e_{i}\right\}_{i=1}^{n+r+1}$ represent the set of polynomials in $P_{n+r}$ dual to the set of linear functionals

$$
\left\{M^{i}\right\}_{i=1}^{n+r+1}=\left\{\mathcal{Q}^{i}\right\}_{i=1}^{s-1} \cup\left\{e_{z}\right\}_{j=s}^{n+r+1}
$$

i.e., $M^{i} e_{j}=\delta_{i j}$ (kronecker delta) $(i, j=1,2, \cdots, n+r+1)$. For each $j=$ $1,2, \cdots, n+r+1$, let $\widetilde{e}_{j}=e_{j} / \mathscr{L}^{s} e_{j}$ if $\mathscr{L}^{s} e_{j} \neq 0$ and let $\widetilde{e}_{j}=e_{j}$ if $\mathscr{L}^{s} e_{j}=$ 0 . Let $\lambda=\left(\lambda_{s}, \lambda_{s+1}, \cdots, \lambda_{n+r+1}\right)^{T}$ be a column vector of $n+r-s+2$ complex numbers and $\|\lambda\|=\left(\left|\lambda_{s}\right|, \cdots,\left|\lambda_{n+r+1}\right|\right)$. We introduce the partial ordering " $\leq$ " defined by $\left\|\lambda^{*}\right\| \leq\|\lambda\|$ provided $\left|\lambda_{j}^{*}\right| \leq\left|\lambda_{j}\right|, s \leq j \leq n+r+1$, and $\left\|\lambda^{*}\right\|<\|\lambda\|$ provided $\lambda \neq 0$ and either $\lambda_{j}^{*}=0$ or $\left|\lambda_{j}^{*}\right|<\left|\lambda_{j}\right|, s \leq j \leq n$ $+r+1$. Let $Q$ be the unique polynomial in $P_{n+r}$ guaranteed by Haar Assumption II such that $\mathcal{L}^{i} Q=A_{i}(i=1,2, \cdots, s-1)$ and $Q\left(z_{i}\right)=0 \quad(i=s$, $s+1, \cdots, n+r+1)$. Finally, let $F$ be the $(r+1) \times(n+r-s+2)$-matrix where $F_{i j}=\widetilde{e}_{j}^{(n+i)}(0)$ for $i=1, \cdots, r$ and $j=s, \cdots, n+r+1$ and $F_{r+1, j}$ $=\mathscr{L}^{s} \widetilde{e}_{j}, j=s, \cdots, n+r+1$, and let $G$ be the $(r+1)$-column vector

$$
\left(-Q^{(n+1)}(0), \cdots,-Q^{(n+r)}(0), A_{s}-\mathcal{L}^{s} Q\right)^{T} \text {. }
$$

Theorem 2. The set of infrapolynomials on $E_{r}$ with respect to $P_{n}(A)$ is 


$$
\left\{Q+\sum_{j=s}^{n+r+1} \lambda_{j}^{*} \tilde{e}_{j} ; \lambda^{*} \text { yields } \min _{F \lambda=G}\|\lambda\|\right\}
$$

Note 4. The condition $F \lambda=G$ is the requirement that $R_{\lambda}=Q+$ $\sum_{j=s}^{n+r+1} \lambda_{j} \tilde{e}_{j} \in P_{n}$ and that $\mathcal{L}^{s} R_{\lambda}=A_{s^{*}}$ (Note that by virtue of $M^{i} e_{j}=\delta_{i j}$, $\mathcal{L}^{i} R_{\lambda}=A_{i}(i=1,2, \cdots, s-1)$.)

Proof of Theorem 2. If $T \in P_{n}(A)$, consider $T^{*}=T-Q \in P_{n+r^{*}}$ Then $\mathfrak{L}^{i} T^{*}=0(i=1,2, \cdots, s-1)$. Thus

$$
T^{*}=\sum_{j=1}^{n+r+1}\left(M^{j} T^{*}\right) e_{j}=\sum_{j=s}^{n+r+1} T^{*}\left(z_{j}\right) \gamma_{j} \tilde{e}_{j}=\sum_{j=s}^{n+r+1} \lambda_{j}^{*} \tilde{e}_{j},
$$

where $\gamma_{j}=\mathscr{\complement}^{s} e_{j}$ or $\gamma_{j}=1$ and $\lambda_{j}^{*}=T\left(z_{j}\right) \gamma_{j}$. Furthermore, clearly $F \lambda^{*}=$ $G$. Thus $T=Q+\Sigma_{j=s}^{n+r+1} \lambda_{j}^{*} \widetilde{e}_{j}$, where $F \lambda^{*}=G$, indicates an arbitrary element of $P_{n}(A)$. Since $Q\left(z_{i}\right)=0(i=s, s+1, \cdots, n+r+1), T$ is an infrapolynomial precisely when $\lambda^{*}$ yields $\min _{F \lambda=G}\|\lambda\|$. This follows from the definition of an infrapolynomial on $E_{r}$ with respect to $P_{n}(A)$, the definition of $\|\cdot\|$, and the partial ordering.

We will give an example of Theorem 2 in the case $r>0$ (see Example 5), but first we restate this result in the case $r=0$ and then observe that we obtain several known results as special cases of the case $r=0$. Let $\beta=A^{s}-\mathscr{L}^{s} Q$ and let $\Sigma^{\prime} \alpha_{j}=\Sigma_{\Sigma^{s} e_{j} \neq 0} \alpha_{j}$.

Corollary 1. The set of infrapolynomials on $E_{0}$ with respect to $P_{n}(A)$ is

$$
\left\{Q+\beta \sum_{j=s}^{n+1} \lambda_{j}^{\prime} \tilde{e}_{j} ; \sum^{\prime} \lambda_{j}^{\prime}=1 \text { wherein } \lambda_{j}^{\prime} \geq 0\right\}
$$

Proof. In case $r=0, R_{\lambda} \in P_{n}$ and $F \lambda=G$ is the single requirement that

$$
\mathscr{L}^{s} R_{\lambda}=\mathscr{L}^{s} Q+\sum_{j=s}^{n+r+1} \lambda \varrho_{j} \tilde{e}_{j}=\mathscr{L}^{s} Q+\sum_{j=s}^{n+r+1} \lambda_{j}=\mathscr{L}^{s} Q+\beta,
$$

i.e. that $\Sigma^{\prime} \lambda_{j}=\beta$. Then $\min \|\lambda\|$ is yielded precisely whenever $\operatorname{sgn} \lambda_{j}=$ $\operatorname{sgn} \beta$ for all $\lambda_{j}$ occurring in $\Sigma^{\prime} \lambda_{j}$, for otherwise $\tilde{\lambda}<\lambda$, where $\tilde{\lambda}_{j}=$ $\left|\lambda_{j}\right| \beta / \Sigma\left|\lambda_{i}\right|$. The conclusion follows by setting $\lambda_{j}^{\prime}=\lambda_{j} / \beta$, if $\beta \neq 0$.

Example 1 (Fekete [2], Motzkin-Walsh [3]). Let $E=\left\{z_{1}, \cdots, z_{n+1}\right\}$ and $g(z)=\prod_{i=1}^{n+1}\left(z-z_{i}\right)$. Then the set of infrapolynomials on $E$ with leading coefficient 1 is 


$$
\left\{\sum_{j=1}^{n+1} \frac{\lambda_{j} g(z)}{\left(z-z_{j}\right)} ; \lambda_{j} \geq 0, \sum \lambda_{j}=1\right\}
$$

This result follows from Corollary 1 by taking $s=1$ and $\mathcal{L}^{1}=e_{0}^{n} / n !$. (Let $e_{z}^{i}$ denote the functional given by $e_{z}^{i} f=f^{(i)}(z)$.) For it is evident that Haar Assumptions I and II hold and that $\widetilde{e}_{j}(z)=g(z) /\left(z-z_{j}\right), j=1,2, \cdots$, $n+1$. Further, since $Q \in P_{n}$ and $Q\left(z_{i}\right)=0(i=1, \cdots, n+1)$, therefore $Q \equiv 0$, whence $\beta=A^{1}=1$. Finally $\mathcal{Q}^{1} \widetilde{e}_{j} \neq 0, j=1,2, \cdots, n+1$, and the statement of the example is therefore seen to be just the statement of Corollary 1 in this case.

Example 1 is a special case of the following example.

Example 2 (Simple $n$-sequences, Shisha-Walsh [5]). Let $E=\left\{z_{s}, \cdots\right.$, $\left.z_{n+1}\right\}$ and $g(z)=\Pi_{i=s}^{n+1}\left(z-z_{i}\right)$. Let $0 \leq l \leq s$ and $q=n-s+l+1$ and suppose $0 \notin E$ if $0<l$. Then the set of infrapolynomials $\sum_{i=0}^{n} c_{i} z^{i}$ on $E$ with $c_{0}, c_{1}, \cdots, c_{l-1}$ and $c_{q}, c_{q+1}, \cdots, c_{n}$ fixed is

$$
\left\{Q(z)+\beta z^{l} \sum_{j=s}^{n+1} \frac{\lambda_{j} g(z)}{\left(z-z_{j}\right)} ; \lambda_{j} \geq 0, \sum \lambda_{j}=1\right\}
$$

where

$$
Q(z)=Q_{1}(z)+z^{l} L(z), \quad Q_{1}(z)=\sum_{i=0}^{l-1} c_{i} z^{i}+\sum_{i=q+1}^{n} c_{i} z^{i}
$$

$L(z)$ is the $(q-D)^{-}$-degree Lagrange interpolating polynomial such that $L\left(z_{i}\right)$ $=-Q_{1}\left(z_{i}\right) / z_{i}^{l}, i=s, \cdots, n+1$, and $\beta=c_{q}-Q^{(q)}(0) / q !$.

To see that this result follows from Corollary 1 , assume first that $l<s$ and take $\mathcal{L}^{i}=e_{0}{ }^{i} / j_{i}$, where $j_{i}=i-1,1 \leq i \leq l$, and $j_{i}=q-i+s, l<i \leq s$. Then $\widetilde{e}_{j}(z)=z^{l} g(z) /\left(z-z_{j}\right), s \leq j \leq n+1$. Indeed $\widetilde{e}_{j}(z)$ is of degree $q$ with highest coefficient 1 so that $\mathcal{Q}^{s} \widetilde{e}_{j}=\widetilde{e}_{j}{ }^{(q)}(0) / q !=1$. Also, since $\widetilde{e}_{j}(z)=$ $\sum_{i=l}^{q} a_{i}^{j} z^{i}$, we have $\mathcal{Q}^{i} \widetilde{e}_{j}=0 \quad(1 \leq i \leq s-1), s \leq j \leq n+1$. Furthermore it is immediately checked that the condition $0 \notin E$, if $0<l$, insures that the Haar Assumptions I and II hold. Thus, we again have that the statement of the example is just the statement of Corollary 1 specified to this case.

In the case $l=s$, i.e. $q=n+1$, apply Corollary 1 to $P_{n+1}\left(A^{\prime}\right)$, where $A^{\prime}=A_{1}, A_{2}, \cdots, A_{s}, 0$. Here $\mathcal{L}^{i}=e_{0}^{i-1} /(i-1) !, 1 \leq i \leq s$. Define also $\mathcal{2}^{s+1}=e_{0}^{n+1} /(n+1) !$. The argument is analogous to that in the case $l<s$. (Note that $c_{q}=c_{n+1}=0$.)

Example 2 with $l=s$ is a special case of the following example.

Example 3 (Infrapolynomials with prescribed values at given points, Walsh-Shisha [6]). Let $E=\left\{z_{s}, \cdots, z_{n+1}\right\}$, let $\xi_{1}, \xi_{2}, \cdots, \xi_{k}$ be distinct 
points of $\mathcal{C}_{z}$, and for each $j=1,2, \cdots, k$, let there be given complex values $w_{j}^{(0)}, w_{j}^{(1)}, \cdots, w_{j}^{\left(m_{j}\right)}$, where $\sum_{j=1}^{k}\left(m_{j}+1\right)=s$. Let

$$
g(z)=\prod_{i=s}^{n+1}\left(z-z_{i}\right) \quad \text { and } \quad h(z)=\prod_{j=1}^{k}\left(z-z_{j}\right)^{m_{j}+1} .
$$

Then the set of infrapolynomials $p(z)=\Sigma_{i=0}^{n} c_{i} z^{i}$ on $E$ with $p^{(\nu)}\left(\xi_{j}\right)=w_{j}^{(\nu)}$, $\nu=0,1, \cdots, m_{j}, j=1,2, \cdots, k$, is

$$
\left\{Q(z)+\beta h(z) \sum_{j=s}^{n+1} \frac{\lambda_{j} q(z)}{\left(z-z_{j}\right)} ; \lambda_{j} \geq 0, \sum \lambda_{j}=1\right\},
$$

where $Q(z)$ is the unique $(n+1)$ st degree polynomial such that $Q^{(\nu)}\left(\xi_{j}\right)=$ $w_{j}^{(\nu)}$ and $Q\left(z_{i}\right)=0, s \leq i \leq n+1$, and $\beta=-Q^{(n+1)}(0) /(n+1) !$.

To see that this result follows from Corollary 1, argue as in Example 2 (where $l=s$ ), observing that $\tilde{e}_{j}(z)=h(z) g(z) /\left(z-z_{j}\right), s \leq j \leq n+1$.

Just as Example 3 follows from Corollary 1 analogously as in Example 2 (with $l=s$ ), the following example follows from Corollary 1 analogously as in Example 2 (with $l<s$ ).

Example 4. Consider the situation of Example 3 except that $\sum_{j=1}^{k}\left(m_{j}+1\right)$ $=t<s$. Then the set of infrapolynomials $p(z)=\Sigma_{i=0}^{n} c_{i} z^{i}$ on $E$ with $p^{(\nu)}\left(\xi_{j}\right)$ $=w_{j}^{(\nu)}, \nu=0,1, \cdots, m_{j}, j=1,2, \cdots, k$, and with $c_{q}, \cdots, c_{n}$ prescribed (where $q=n-s+t^{\prime}+1$ ) is

$$
\left\{Q(z)+\beta h(z) \sum_{j=s}^{n+1} \frac{\lambda_{j} g(z)}{\left(z-z_{j}\right)} ; \lambda_{j} \geq 0, \sum \lambda_{j}=1\right\},
$$

where $Q(z)$ is the unique element of $P_{n}$ such that $Q^{(\nu)}\left(\xi_{j}\right)=w_{j}^{(\nu)} \quad(\nu=0$, $\left.1, \cdots, m_{j}\right), Q^{(i)}(0) / i !=c_{i}(q+1 \leq i \leq n), Q\left(z_{i}\right)=0 \quad(i=s, \cdots, n+1)$, and $\beta=c_{q}-Q^{(q)}(0) / q !$.

The following are examples of Theorem 2 in case $r \geq 0$.

Example 5. Let $E=\left\{z_{1}, \cdots, z_{n+r+1}\right\}$ and $g(z)=\Pi_{i=1}^{n+r+1}\left(z-z_{i}\right)$. For each $k=0,1, \cdots, r$, let $\sigma_{j}^{k}$ be the $k$ th symmetric function $\left(\sigma_{j}^{0}=1\right)$ of the $n+r$ numbers in $E-\{z, j=1, \cdots, n+r+1$. Then the set of $n$th degree infrapolynomials on $E$ with leading coefficient 1 is

$$
\begin{aligned}
\left\{\sum_{j=1}^{n+r+1} \frac{\lambda_{j} g(z)}{\left(z-z_{j}\right)} ; \lambda \text { is minimal subject to } \sum \lambda_{j}=1\right. \text { and } \\
\left.\sum \frac{\lambda_{j} \sigma_{j}^{k}}{\sigma_{j}^{r}}=0(k=0,1, \cdots, r-1)\right\} .
\end{aligned}
$$


This follows immediately from Example 1 and from an analysis of the condition $F \lambda=G$ in this case.

Note 5. Notice that the set of infrapolynomials in Example 5 includes

$$
\left\{\sum_{j=1}^{n+r+1} \frac{\lambda_{j} g(z)}{\left(z-z_{j}\right)} ; \lambda_{j} \geq 0, \sum \lambda_{j}=1, \sum \frac{\lambda_{j} \sigma_{j}^{k}}{\sigma_{j}^{r}}=0 \quad(k=0,1, \cdots, r-1)\right\} .
$$

We tum now to some results on the location of zeros of infrapolynomials on $E_{r}$ with respect to $P_{n}(A)$. The situation is that of Theorem 2. Let $\left\{\lambda^{*}\right\}=$ $\left\{\lambda^{*} ; \lambda^{*}\right.$ yields $\left.\min _{F \lambda=G}\|\lambda\|\right\}$, so that $\left\{\lambda^{*}\right\}$ is a subset of complex $(n+r-s$ $+2)$-space.

Theorem 3. In Theorem 2, if $\left\{\lambda^{*}\right\}$ is bounded, and if $\operatorname{deg} Q>\max \operatorname{deg} \widetilde{e}_{j}$, $s \leq j \leq n+r+1$, then $\exists H<\infty$ such that, if $I\left(z_{0}\right)=0$ for some infrapolynomial 1 , then $\left|z_{0}\right| \leq H$.

Proof. $I(z)=Q(z)+P(z)$, where $Q(z)=a_{q} z^{q}+a_{q-1} z^{q-1}+\cdots, P(z)=$ $b_{p} z^{p}+b_{p-1} z^{p-1}+\cdots$ and $a_{q} b_{p} \neq 0$. Since $\left\{\lambda^{*}\right\}$ is bounded, all the coefficients $b_{j}$ of $P(z)$ are bounded in absolute value by $|\beta|(n+1) m M$, where $m=$ $\max _{\lambda^{*}, j}\left|\lambda_{j}^{*}\right|$ and $M$ is the maximum modulus of all the coefficients of the $\tilde{e}_{j}$. Thus $0=I\left(z_{0}\right)=Q\left(z_{0}\right)+P\left(z_{0}\right)$, whence

$$
\begin{array}{r}
z_{0}=a_{q}^{-1}\left(-a_{q-1}-a_{q-2} z_{0}^{-1}-\cdots-a_{0} z_{0}^{-(q-1)}-b_{p_{0}} z_{0}^{-(q-p-1)}\right. \\
\left.-\cdots-b_{0} z_{0}^{-(q-1)}\right) .
\end{array}
$$

Since $q>p \geq 0$, we can set

$$
H=\max \left\{1,\left|a_{q}\right|^{-1}\left(\sum_{0}^{q-1}\left|a_{i}\right|+\sum_{0}^{p}\left|b_{j}\right|\right)\right\} .
$$

Corollary 2. In Corollary 1 , if $\mathfrak{Q}^{s} e_{j} \neq 0(s \leq j \leq n+1)$ and if $\operatorname{deg} Q>$ $\max \operatorname{deg} \widetilde{e}_{j}, s \leq j \leq n+1$, then $H<\infty$ such that, if $I\left(z_{0}\right)=0$ for some infrapolynomial 1 , then $\left|z_{0}\right| \leq H$.

Proof. Clearly from $(*),\left|\lambda_{j}^{*}\right|=|\beta|\left|\lambda_{j}^{\prime}\right| \leq|\beta|, s \leq j \leq n+1$, and so $\left\{\lambda^{*}\right\}$ is bounded. The conclusion then follows from Theorem 3.

Let smp $R$ denote the smallest power of $z$ occurring in $R$.

Theorem 4. In Theorem 2, if $\left\{\lambda^{*}\right\}$ is bounded and if $\operatorname{smp} Q<\min \operatorname{smp} \widetilde{e}_{j}$, $s \leq j \leq n+r+1$, then $\exists h>0$ such that, if $I\left(z_{0}\right)=0$ for some infrapolynomial $I$, then $\left|z_{0}\right| \geq h$.

Proof. $I(z)=Q(z)+P(z)$, where $Q(z)=a_{m} z^{m}+a_{m+1} z^{m+1}+\cdots$ and 
$P(z)=b_{p} z^{p}+b_{p+1} z^{p+1}+\cdots+b_{q} z^{q}$. Again, since $\left\{\lambda^{*}\right\}$ is bounded, all the coefficients $b_{j}$ are bounded in absolute value. Thus $0=I\left(z_{0}\right)=Q\left(z_{0}\right)+$ $P\left(z_{0}\right)$, whence

$$
1=a_{m}^{-1}\left(-a_{m+1} z_{0}-\cdots-a_{q} z_{0}^{q-m}-b_{p} z_{0}^{p-m}-\cdots-b_{q} z_{0}^{q-m}\right)=t\left(z_{0}\right) .
$$

Since $q \geq p>m \geq 0, \exists h>0$ such that $|t(z)| \leq 1 / 2$ if $|z| \leq h$.

Note 6. If either $s=1$ or $A_{i}=0(1 \leq i \leq s-1)$, then $Q \equiv 0$ and the hypotheses of Theorem 4 reduce to $\left\{\lambda^{*}\right\}$ being bounded.

Corollary 3. In Corollary 1, if $\mathcal{Q}^{s} e_{j} \neq 0(s \leq j \leq n+1)$ and if $\operatorname{smp} Q<$ min smp $\widetilde{e}_{j}, s \leq j \leq n+1$, then $\exists h>0$ such that, if $I\left(z_{0}\right)=0$ for some infrapolynomial $I$, then $\left|z_{0}\right| \geq h$.

Note 7. In the case of Example 2 above (simple $n$-sequences), Corollaries 2 and 3 of this paper give Theorem 6 of [5].

Remark. Theorems 1 and 2 and Corollary 1 are valid if, for each $m=$ $0,1,2, \cdots, P_{m}$ is replaced by $V_{m}$, an arbitrary $(m+1)$-dimensional subspace of $C\left(\mathcal{C}_{z}\right)^{m}$ satisfying $V_{m} \subset V_{m+1}$, and $f^{(q)}(0)$ is replaced by $K_{q} f$, where $K_{1}, K_{2}, \cdots$ is a sequence of linear functionals defined on $\bigcup_{V_{m}}$ such that $V_{m}=V_{m+1} \cap$ nullspace of $K_{m+1}$. Also Theorems 3 and 4 and their corollaries have obvious analogues in this general situation.

The author is grateful to Dr. Oved Shisha for his encouragement in connection with this paper.

\section{REFERENCES}

1. B. L. Chalmers, On weak underpolynomials of generalized infrapolynomials, J. Approximation Theory 7 (1973), 391-394.

2. M. Fekete, On the structure of extremal polynomials, Proc. Nat. Acad. Sci. U. S. A. 37 (1951), 95-103. MR 13, 32.

3. T. S. Motzkin and J. L. Walsh, Underpolynomials and infrapolynomials, Illinois J. Math. 1 (1957), 406-426. MR 19, 643.

4. O. Shisha, On the structure of infrapolynomials with prescribed coefficients, Pacific J. Math. 14 (1964), 1039-1051. MR 30 \#231.

5. O. Shisha and J. L. Walsh, The zeros of infrapolynomials with some prescribed coefficients, J. Analyse Math. 9 (1961/62), 111-160. MR 25 \#174.

6. - The zeros of infrapolynomials with prescribed values at given points, Proc. Amer. Math. Soc 14 (1963), 839-844. MR 27 \#3785.

DEPARTMENT OF MATHEMATICS, UNIVERSITY OF CALIFORNIA, RIVERSIDE, CALIFORNIA 92502 